\title{
On the origin of the metallicity gradient in clusters of galaxies
}

\author{
K. Umetsu and M. Hattori
}

Astronomical Institute, Graduate School of Science, Tohoku Iniversity, Aramaki-aza-aoba. 1oba-ku, Sendai, Miyagi 980-8578, Japan

\section{ABSTRACT}

The cosmological origin of a large-scale gradient in the metallicity of the intra-cluster medium (ICM) discovered by ASCA has been examined. If the metal enrichment of the ICM completed before the gravitational collapse of the cluster, it is expected that the local galaxy-formation efficiency in the protocluster is imprinted as the local metallicity of the ICM. The galaxy-formation efficiency might be higher in the central region of the cluster than in its outskirts since the cluster-scale density fluctuation is higher and acts more efficient for pushing galaxy-scale density fluctuations up nearer to the cluster center. We extend the Press-Schechter theory to take into account the cluster environmental effects on the mass function of the virialized objects, derive an analytic formula to calculate the local galaxy-formation efficiency, and obtain the expected cosmological metallicity gradient in the ICM. Our results show, however, no significant metallicity gradient for our rich- nor poor-cluster models. On the otherhand, our canonical model for the XD cluster show a remarkable gradient in the metallicity distribution.

(C) 2000 COSPAR. Published by Elsevier Science Ltd.

\section{INTRODUCTION}

$\mathrm{X}$-ray observations of galaxy clusters show that they have a significant amount of hot gas, which are called intra-cluster medium (hereafter ICM). The ICM contains a large amount of heavy elements, e.g., $\mathrm{O}, \mathrm{Ne}, \mathrm{Mg}, \mathrm{Si}, \mathrm{S}, \mathrm{Ar}, \mathrm{Ca}$, and $\mathrm{Fe}$. It has been shown by X-ray observations that the Fe abundance is roughly constant from dynamically-young to dynamically-old clusters. The measurement of the iron mass to optical-luminosity ratio suggests that the metals in the ICM are originated from elliptical galaxies (Arnaud et al. 1992). The detection of an overabundance of $\alpha$-elements with ASCA suggests that the metals in the ICM might come mainly from Type II SNe (Loewenstein and Mushotzky 1996; Mushotzky et al. 1996). Thus one of the possible mechanisms for the metal enrichment of the ICM is a wind from proto-galaxies derived by Type II SNe explosion in their early evolutionary phase (hereafter early galactic wind). In a hierarchical universe, it has been shown that the epoch of galaxy formation is earlier than that of cluster formation. We define the galaxy-formation efficiency $\epsilon_{g a l}$ in some region as $\epsilon_{\mathrm{gal}}=M_{\text {star }} /\left(M_{\mathrm{gas}}+M_{\mathrm{star}}\right)=M_{\mathrm{gal}} / M_{\mathrm{tot}}$, where $M_{\text {gas }}$ is the gas mass, $M_{\text {star }}$ is the mass of stars, $M_{\text {gal }}$ is the mass of galaxies, and $M_{\text {tot }}$ is the total mass in that region. Then the metallicity correlates positively with $\epsilon_{\text {gal }}$ and reflects it strongly (see Eq. (2)). If the metal enrichment of the ICM completes before the gravitational collapse of the cluster, it is expected that the galaxy-formation efficiency in the protocluster is imprinted as the metallicity of the ICM. The effects of iron sedimentation is negligible (Rephaeli 1978), and that of iron diffusion is also negligible (Ezawa et al. 1997). Therefore the distribution of the galaxy-formation efficiency might be preserved and observed as the metallicity distribution in the ICM. We extend the Press-Schechter theory to take into account the cluster environmental effects on the mass function of the virialized objects and derive an analytic formula to calculate the local galaxy-formation efficiency in a hierarchical universe. Then we construct a simple analytic model for the metal injection 
and obtain the expected cosmological metallicity distribution in the ICM.

\section{Environmental effects on the galaxy formation efficiency}

We assume that the density contrast $\delta(\vec{x}):=[\rho(\vec{x})-\bar{\rho}] / \bar{\rho}$, linearly extrapolated to the present epoch, is a. random Gaussian field completely determined by its power spectrum, where $\vec{x}$ represents a comoving Lagrangian coordinate. Then a density field smoothed with a spherically-symmetric window function $W(k ; R)$ is obtained by $\delta_{R}(\vec{x}):=\int d^{3} k W(k ; R) \hat{\delta}(\vec{k}) \mathrm{e}^{i \vec{k} \cdot \vec{x}} \equiv \delta_{M}(\vec{x})$ with mass $M \sim \bar{\rho} R^{3}$. According to the top-hat spherical model of the gravitational collapse, properties of virialized objects are determined by two parameters: the mass $M$ and its formation epoch $z$. We specify the proto-cluster perturbation centered at $\vec{x}$ supposed to form a cluster at redshift $z_{\mathrm{cl}}$ with mass $M_{\mathrm{cl}}$ by $\vec{\nabla} \delta_{M_{\mathrm{cl}}}(\vec{x})=0$ and $\delta_{M_{\mathrm{cl}}}(\vec{x})=\delta_{\mathrm{c}}\left(z_{\mathrm{cl}}\right)$, where $\delta_{\mathrm{c}}(z)$ is the critical over density; for an Einstein-de Sitter universe, $\delta_{\mathrm{c}}(z)=1.686(1+z)$. We consider the conditional probability distribution $P\left(\delta_{M}(r) \mid \vec{\nabla} \delta_{M_{\mathrm{cl}}}(0)=0, \delta_{M_{\mathrm{cl}}}(0)=\delta_{c}\left(z_{\mathrm{cl}}\right)\right)$ of $\delta_{M}(r) \equiv \delta_{M}(\vec{x}+\vec{r})$ with a mean $\left\langle\delta_{M} \mid M_{\mathrm{cl}}, z_{\mathrm{cl}}, r\right\rangle$ and a variance $\sigma^{2}\left(M \mid M_{\mathrm{cl}}, z_{\mathrm{cl}}, r\right)$, from which we have derived the conditional mass-distribution function for the virialized objects with mass $M$ to $M+d M$ at redshift $z$, depending on the environmental parameters, i.e. the cluster properties, $M_{c l}$ and $z_{\mathrm{cl}}$, and the distance $r$ from the proto-cluster center in Lagrangian space:

$$
\epsilon\left(M, z \mid M_{\mathrm{cl}}, \tilde{z}_{\mathrm{cl}}, r\right) d M=\sqrt{\frac{1}{2 \pi}} \frac{\delta_{c}(z)-\left\langle\delta_{M} \mid M_{\mathrm{cl}}, z_{\mathrm{cl}}, r\right\rangle}{\left[\sigma^{2}\left(M \mid M_{\mathrm{cl}}, z_{\mathrm{cl}}, r\right)\right]^{3 / 2}} \exp \left\{\frac{\left.\left[\delta_{c}(z)-\left\langle\delta_{M}\right| M_{\mathrm{cl}}, z_{\mathrm{cl}}, r\right)\right]^{2}}{2 \sigma^{2}\left(M \mid M_{\mathrm{cl}}, z_{\mathrm{cl}}, r\right)}\right\}\left|\frac{\partial \sigma^{2}(M)}{\partial M}\right| d M
$$

with $\left\langle\delta_{M} \mid M_{\mathrm{cl}}, z_{\mathrm{cl}}, r\right\rangle=\left[\xi(r) / \sigma^{2}(M)\right] \delta_{\mathrm{c}}\left(z_{\mathrm{cl}}\right)$ and $\sigma^{2}\left(M \mid M_{\mathrm{cl}}, z_{\mathrm{cl}}, r\right)=\sigma^{2}(M)-\xi^{2}(r) / \sigma^{2}\left(M_{\mathrm{cl}}\right)-\left[\xi^{\prime}(r)\right]^{2} / \alpha$. Here $\sigma^{2}(M), \xi(r)$, and $\alpha$ are defined by $\sigma^{2}(M)=\left\langle\delta_{M}^{2}(\vec{x})\right\rangle, \xi(r)=\left\langle\delta_{M_{\mathrm{cl}}}(\vec{x}) \delta_{M}(\vec{x}+\vec{r})\right\rangle$, and $\alpha=$ $\left\langle\left[\vec{\nabla} \delta_{M_{\mathrm{cl}}}(\vec{x})\right]^{2}\right\rangle / 3$. Using this conditional mass-distribution function, we can calculate the local galaxyformation efficiency $\epsilon_{\text {gal }}(r)$ depending on the cluster-environmental parameters.

\section{Relation between galaxy formation efficiency and metallicity}

We assume that the metal enrichment of the ICM completes before the virialization of the cluster. Here we adopt the Type II SNe-driven galactic wind as a mechanism for the metal enrichment of the ICM. For analytical simplicity, several assumptions are made: (i) The early galactic wind occurs at a single characteristic redshift $z_{\mathrm{GW}}>z_{\mathrm{cl}}$. (ii) All galaxies with mass range from $M_{\text {low }}$ to $M_{\text {up }}$ eject metals at redshift $z_{\mathrm{GW}}$. (iii) The mass fractions $f_{\mathrm{Fe}}=M_{\mathrm{Fe}}^{\mathrm{ej}} / M_{\mathrm{gas}}$ and $f_{\mathrm{gas}}=M_{\mathrm{gas}}^{\mathrm{ej}} / M_{\mathrm{gas}}$ are independent of the galaxy mass $M_{\mathrm{gal}}$. Here $M_{\mathrm{gas}}$ is the total gas mass which each galaxy contains initially. $M_{\mathrm{Fe}}^{\mathrm{ej}}$ and $M_{\mathrm{gas}}^{\mathrm{ej}}$ are the iron mass and the gas mass, respectively, ejected through the early galactic wind. Concerning the estimation of $f_{\text {gas }}$ and $f_{\mathrm{Fe}}$, we use Salpeter's initial mass function and data of metallicity of Type II SNe ejecta (Nomoto et al.). Using our model, we obtain $f_{\mathrm{Fe}} \simeq 3,33 \times 10^{-4}$ and $f_{\mathrm{gas}} \simeq 7.31 \times 10^{-2}$. Here we assume the following: (iv) All gas converts into stars instantaneously. (v) Stars are born with mass range from $0.05 m_{\odot}$ to $40 m_{\odot}$. (vi) The gas loss occurs in stars with mass greater than $8 m_{\odot}$, and the remnant is $1.4 m_{\odot}$. (vii) All iron products by Type II SNe are injected in to the ICM.

The local galaxy-formation efficiency $\epsilon_{\text {gal }}(r)$ is related to the local iron abundance $Z_{\mathrm{Fe}}(r)$ of the ICM in the following way:

$$
Z_{\mathrm{Fe}}(r)=\frac{\frac{1}{56} f_{\mathrm{Fe}} \epsilon_{\mathrm{gal}}(r)}{\frac{9}{13}\left[1-\epsilon_{\mathrm{gal}}(r)+f_{\mathrm{gas}} \epsilon_{\mathrm{gal}}(r)\right]}
$$

We use a value of $Z_{\odot}=1.67 \times 10^{-5}$ for the solar iron abundance (Anders et al. 1989). Then we have $Z_{\mathrm{Fe}} / Z_{\odot} \simeq 2.5$ with $\epsilon_{\mathrm{gal}}=1$. For a background cosmology, we adopt an Einstein-de Sitter universe with the standard biased CDM spectrum $\left(\Omega_{0}=1, h_{50}=H_{0} / 50 \mathrm{~km} \mathrm{~s}^{-1} \mathrm{Mpc}^{-1}=1, \Omega_{\mathrm{b}}=0.05\right.$, and $\left.\sigma_{8}=0.63\right)$. First, we calculate the local galaxy-formation efficiency, averaged over the ensemble 
of the proto-cluster density perturbations supposed to form a cluster with mass $M_{\mathrm{cl}}$ at redshift $z_{\mathrm{cl}}=$ 0 , by $\epsilon_{\mathrm{gal}}(r)=\int_{M_{\text {low }}}^{M_{\mathrm{p}}} d M \epsilon\left(M, z_{\mathrm{GW}} \mid M_{\mathrm{cl}}, z_{\mathrm{cl}}, r\right)$. We consider two cluster masses $M_{\mathrm{cl}}=1.0 \times 10^{14} M_{\odot}$ (corresponding to a virial radius of $1.2 \mathrm{Mpc}$ ) and $1.0 \times 10^{15} M_{\odot}$ (corresponding to a virial radius of 2.7 $\mathrm{Mpc}$ ), representative of poor and rich clusters, respectively. Then unknown parameters are the lower and the upper mass of the metal injector $M_{\text {low }}$ and $M_{\mathrm{up}}$, respectively, and the redshift $z_{\mathrm{GW}}$ at which the early galactic wind occurs. Since typical mass of the cD galaxy is about $10^{13} M_{\odot}$, we set $M_{\text {up }}=1.0 \times 10^{13} M_{\odot}$. Thus two parameters $M_{\text {low }}$ and $z_{\mathrm{GW}}$ are left free in our calculation. Next, we calculate the radial profile of the iron abundance in the ICM assuming that the ICM do not cross each other during the cluster evolution, i.e., that the radial distribution of the local galaxy-formation efficiency $\epsilon_{\mathrm{gal}}(r)$ at redshift $z_{\mathrm{GW}}$ determines that of the iron abundance at redshift $z_{\mathrm{cl}}=0$. Since the local galaxy-formation efficiency derived by extending the Press-Schechter theory is expressed with the radius in Lagrangian coordinate, we have to transform the Lagrangian coordinate into the Eulerian one. For this purpose, we employ the "universal" CDM profile for the mass profile of the virialized cluster (Navarro, Frenk, and White 1997) and relate the Lagrangian radius with the Eulerian one assuming that the mass contained within each shell does not change with time. We set $c=10$ in every case, where $c$ is the concentration parameter of the universal CDM profile. We have calculated the local galaxy-formation efficiency $\epsilon_{\text {gal }}$ for $M_{\text {low }}=1.0 \times 10^{9} M_{\odot}$ and $1.0 \times 10^{11} M_{\odot}$ at redshifts $z_{\mathrm{GW}}=1,3$, and 5 . We plot our calculations of iron abundance as a function of Eulerian radius for $M_{\text {low }}=1.0 \times 10^{11} M_{\odot}$ (see Figs. 1 and 2).

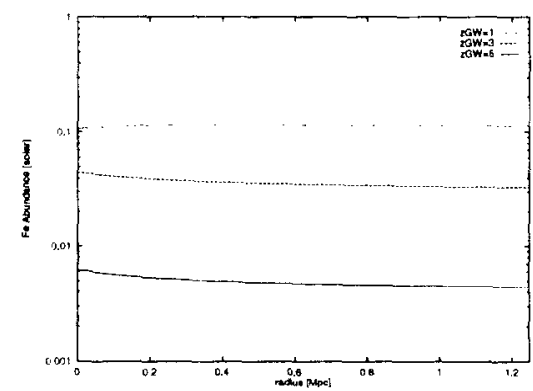

Figure 1: The Fe abundance as a function of radius, calculated for clusters which virialize at $z_{\mathrm{cl}}=0$ with $M_{\mathrm{cl}}=10^{14} M_{\odot}$.

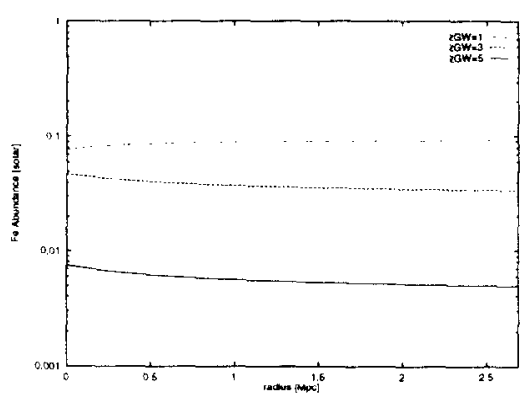

Figure 2: The Fe abundance as a function of radius, calculated for clusters which virialize at $z_{\mathrm{cl}}=0$ with $M_{\mathrm{cl}}=10^{15} M_{\odot}$.

\section{Results and Discussion}

The main results are summarized as follows: (1) No significant metallicity gradient can be seen in any case. (2) There are little differences between two clusters especially in cases with $M_{\text {low }}=1.0 \times 10^{9} M_{\odot}$. (3) In models with $M_{\text {low }}=1.0 \times 10^{11} M_{\odot}$, the galaxy formation is more efficient in poor clusters than in rich clusters. (4) The depression of $\epsilon_{\text {gal }}(r)$ near the cluster center is found in the result with $z_{G W}=1$. We consider that the result (1) is due to the variation of the proto-cluster perturbation that will virialize at redshift $z_{c l}$ with mass $M_{c l}$. For example, a bimodal cluster could not have a high galaxy-formation efficiency near the proto-cluster center in Lagrangian space. As a consequence, the radial profile of $\epsilon_{\text {gal }}$ averaged over clusters including bimodal ones may become flatter than that averaged over clusters excluding them. The results (2) and (3) indicate that there exists a greater statistical correlation between galaxy and poor-cluster masses than between galaxy and rich-cluster masses and that such correlation is weaker for less massive galaxies, consistent with the result of David and Blumenthal (1992). Concerning the result (4), the depression of $\epsilon_{\text {gal }}(r)$ occurs since the matter in the central region of the proto-cluster collapses into halos with mass greater than $M_{\mathrm{up}}=1.0 \times 10^{13} M_{\odot}$ at this epoch. These theoretical results should be compared with the radial distribution of observed Fe abundance averaged over an ensemble of the clusters with identical ICM temperature. Using the ASCA results, Fukazawa (1997) averaged Fe abundances of clusters at various radii within $1 h_{50}^{-1} \mathrm{Mpc}$ separately for XD and non-XD clusters. Non-XD 
clusters show little Fe-abundance gradient, while XD clusters show the abundance concentration in the cluster center. These results encourage us to construct a model of the local galaxy-formation efficiency for XD-clusters. We consider the proto-cluster density perturbation that will form a $\mathrm{cD}$ galaxy at redshift $z_{\mathrm{cD}}$ with mass $M_{\mathrm{cD}}=1.0 \times 10^{13} M_{\odot}$ in the proto-cluster center and will virialize at redshift $z_{\mathrm{cl}}=0$ with mass $M_{\mathrm{cl}}=1.0 \times 10^{15} M_{\odot}$. We take the redshift $z_{\mathrm{cD}}$ of the cD-galaxy formation higher than unity (Hattori et al. 1997; Aragón-Salamanca et al. 1998) and calculate the radial profile of the Fe abundance in the same way as described in Section 3. The results for our canonical model of the Fe abundance for XD clusters are shown in Figs 3 and 4. Theoretical predictions of the metallicity profile for XD clusters show a considerable Fe concentration toward the cluster center, similar to the result for XD clusters by Fukazawa (1997).

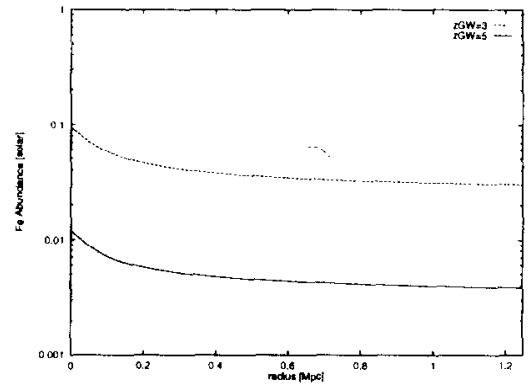

Figure 3: The Fe abundance as a function of radius, calculated for our model of the XD clusters which virialize at $z_{\mathrm{cl}}=0$ with $M_{\mathrm{cl}}=10^{14} M_{\odot \cdot)}$. We use $M_{\text {low }}=10^{11} M_{\odot}$ for a lower mass of the metal injector.

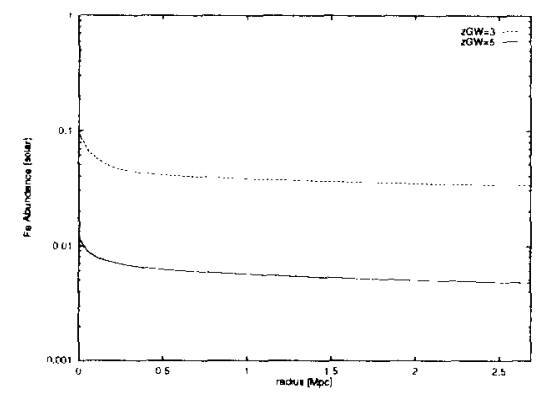

Figure 4: The Fe abundance as a function of radius, calculated for our model of the XD clusters which virialize at $z_{\mathrm{cl}}=0$ with $M_{\mathrm{cl}}=10^{15} M_{\odot}$. We use $M_{\text {low }}=10^{11} M_{\odot}$ for a lower mass of the metal injector.

\section{REFERENCES}

Anders, E. and Grevesse, N., Abundances of the elements-meteoritic and solar. Geochim. Cosmochim. Acta, 53, 197 (1989).

Aragón-Salamanca, A., Baugh, C. M., and Kauffmann, C., Mon. Not. R. Astran. Soc., 297, 427 (1998).

Arnaud, M., Rothenflug, R., Boullade, O., Vigroux, L., Vangioni-Flam, E., Astron. Astrophys., 254, 49 (1992).

Bond, J. R., Cole, S., Efstathiou, G., and Kaiser, N., Astrophys. J., 379, 440 (1991).

David, L. P. and Blumenthal, G. R., Astrophys. J., 389, 510 (1992).

Ezawa, H., Fukazawa, Y., Makishima, K., Ohashi, T., Takahara, F., Xu, H., and Yamasaki, Y., Astrophys. .J., 490, L33 (1997).

Fukazawa, Y., X-Ray Study of Metal Enrichment Processes of Hot Gas in Clusters of Galaxies, Ph.D. Thesis, University of Tokyo (1997).

Hattori, M., Ikebe, Y., Asaoka, I., Takeshima, T., Böohringer, H., Mihara, T., Neumann, D. M., Schindler, S., Tsuru, T., and Tamura, T., Nature, 388, 6638 (1997).

Loewenstein, M. and Mushotzky, R. F., Astrophys. J., 466, 695 (1996).

Mushotzky, R., Loewenstein, M., Arnaud, K. A., Tamura, T., Fukazawa, Y., and Matsushita, K., Astrophys. J., 466, 686 (1996).

Navarro, J. F., Frenk, C. S., and White, S. D. M., Astrophys. J. , 490, 493 (1997).

Nomoto, K. and Tsujimoto, T., private communication.

Preess, W. H. and Schechter, P., Astrophys. J., 187, 425 (1974).

Rephaeli, Y., Astrophys. J., 225, 33 (1978). 